\title{
Correction: RKIP regulates CCL5 expression to inhibit breast cancer invasion and metastasis by controlling macrophage infiltration
}

\section{Ila Datar, Xiaoliang Qiu, Hong Zhi Ma, Miranda Yeung, Shweta Aras, Ivana de la Serna, Fahd Al-Mulla, Jean Paul Thiery, Robert Trumbly, Fan Xuan, Hongjuan Cui, Kam C. Yeung}

Present: Due to an error made by the authors while submitting a revision, Dr. Tuan Zea Tan was omitted from the list of authors.

Corrected: Correct author list can be found below. Authors sincerely apologize for this oversight.

Original article: Oncotarget. 2015; 6(36): 39050-61. doi: 10.18632/oncotarget.5176.

\footnotetext{
Ila Datar ${ }^{1}$, Xiaoliang Qiü ${ }^{1}$, Hong Zhi Ma ${ }^{1}$, Miranda Yeung ${ }^{1}$, Shweta Aras ${ }^{1}$, Ivana de la Serna ${ }^{1}$, Fahd Al-Mulla², Tuan Zea Tan ${ }^{3}$, Jean Paul Thiery ${ }^{3,4}$, Robert Trumbly ${ }^{1}$, Fan Xuan ${ }^{5}$, Hongjuan Cui ${ }^{5}$ and Kam C. Yeung ${ }^{1}$

${ }^{1}$ Department of Biochemistry and Cancer Biology, University of Toledo, College of Medicine, Health Science Campus, Toledo, $\mathrm{OH}$, USA

2 Kuwait University, Faculty of Medicine, Safat, Kuwait

${ }^{3}$ Cancer Science Institute of Singapore National University of Singapore, Center for Translational Medicine, Singapore, Singapore

${ }^{4}$ Department of Biochemistry, Yong Loo Lin School of Medicine, National University of Singapore, Singapore, Singapore

${ }^{5}$ State Key Laboratory Of Silkworm Genome Biology, Chongqing, China
} 